\title{
On a Flow-Shop Scheduling Problem with Fuzzy Pentagonal Processing Time
}

\author{
Majed G. Alharbi ${ }^{1}{ }^{1}$ and Hamiden Abd El-Wahed Khalifa $\mathbb{D}^{2,3}$ \\ ${ }^{1}$ Department of Mathematics, College of Science and Arts, Qassim University, Methnab, Buridah, Saudi Arabia \\ ${ }^{2}$ Operations Research Department, Faculty of Graduate Studies for Statistical Research, Cairo University, Giza, Egypt \\ ${ }^{3}$ Mathematics Department, College of Science and Arts, Al-Badaya, Qassim University, Buridah, Saudi Arabia
}

Correspondence should be addressed to Majed G. Alharbi; dr.majed.qu@gmail.com

Received 17 December 2020; Revised 4 April 2021; Accepted 10 April 2021; Published 28 April 2021

Academic Editor: Basil Papadopoulos

Copyright (C) 2021 Majed G. Alharbi and Hamiden Abd El-Wahed Khalifa. This is an open access article distributed under the Creative Commons Attribution License, which permits unrestricted use, distribution, and reproduction in any medium, provided the original work is properly cited.

\begin{abstract}
Scheduling involves planning and arranging jobs across a coordinated set of events to satisfy the customer's demands. In this article, we present a simple approach for the flow-shop (FS) scheduling problem under fuzzy environment in which processing time of jobs are represented by pentagonal fuzzy numbers. This study is intended to reduce the rental cost of the machine in compliance with the rental policy. The fuzzy FS scheduling problem is solved without converting the processing time into its equivalent crisp numbers using a robust ranking technique and a fuzzy arithmetic pentagonal fuzzy numbers. A numerical illustration indicates that the approach is workable, accurate, and relevant.
\end{abstract}

\section{Introduction}

Scheduling requires a variety of activities to accomplish a particular purpose with time and budget. The job schedule and management of its flows through a planning step are the most fundamental facets of any industrial production procedure. The management of a certain number of machines or facilities for a certain number of tasks or employment is one difficulty. The control of staff in any manner poses a largely unresolved obstacle to accomplish a certain objective. In planning a production or plan, a decision maker has difficulties encouraging prompt implementation and reducing demands such as flow times or completion periods. The scheduler's goal is to set starting times to achieve maximum performance subject to energy and technological constraints. The problem with flowshop preparation is that the simplistic version of which all staffs travel in the same sequence on all machinery is one of the most critical issues in production management. To minimize a machine's completion time, Argawal et al. [1] encountered three FS device scheduling problems. Vahedi-Nouri et al. [2] presented a more broad version of the FS strategy to minimize the average flow rate. In order to optimize the publication time, Ren et al. [3] explored the topic of FS programming. Laribi et al. [4] have introduced a mathematical model for two FS-limited machines that address FS time reduction problems where renewables are not constrained. Yazdani and Naderi [5] considered the scheduling problem with no-idle hybrid flow-shop and applied a mixed integer linear programming to formulate the problem. Qu et al. [6] designed a flower pollination algorithm based on the hormone modulation mechanism for nowait flow-shop scheduling problem, where the method uses available neighborhood search strategy based on dynamic selfadaptive variable work piece in the local search.

This would be made possible by a blueprint for several research fields such as computer analysis and organizational science. Decision-making in a fuzzy environment, developed by Bellman and Zadeh [7], has an improvement and a great help in the management decision problems. Fuzzy numerical data can be represented by means of fuzzy subsets of the real line, known as fuzzy numbers. Dubois and Prade [8] spread the expansion of algebraic operations into fuzzy numbers by a fuzzy hypothesis of real numbers. Panda and Pal developed the theory of pentagonal andantes fuzzy numbers (2016). Chakraborty et al. [9] extended the properties of the pentagonal fuzzy number to 
interval-value fuzzy and pentagonal fuzzy numbers. Many scientists $[10,11]$ were involved in the problem of FS preparation. A method containing a variety of statistical and fuzzy flow-shop sets was introduced by Yao and Lin [12]. Hejari et al. [13] enhanced McCahon and Lee's [14] algorithm for work sequence with a fuzzy processing time (1990). For a smooth processing time, Ishibuchi and Lee established FS scheduling problem [15]. Temiz and Erol [16] also employed the fuzzy scheduling principle of the FS, updated the branch-and-bound of Ignall and Schrage [17], and reassigned the Ignall and Schrage algorithm to three FS frame problems. Gupta et al. [18] applied a heuristic algorithm for minimizing the rental cost of the machines for a specially structured three-stage flow-shop scheduling where triangular fuzzy numbers represent the processing times of the machine. Neira et al. [19] provided a general overview of the FS scheduling problem under uncertainties and its role in production logistics to draw up opportunities for further research. Komaki et al. [20] conducted a joint survey of their templates for assembly flow shops. Janaki and Ismail [21] explored the interval, modeling work using an efficient and heuristic make-up approach. Khalifa [2] analyzed the singlemachine preparation issue in a fizzy dates setting. Khalifa et al. [23] proposed a fuzzy binding approach for solving constrained flow-shop scheduling problem without converting the problem into its crisp.

In this paper, we consider the problem of flow-shop scheduling in a fuzzy environment where processing times are represented as pentagonal fuzzy numbers. We investigate a method to find the rental cost of the machines without converting the pentagonal fuzzy numbers into its classical number.

This study aims to minimize the rental costs of the machine as described in the rental policy. The remainder of the article is organized as follows. Section 2 addresses some preliminaries. Section 3 includes some fundamental assumptions and concepts that are central to the problem. Section 4 contains the pentagonal fuzzy number processing times' assertion of the FS programming problem. Section 5 offers an approach to the problem of fuzzy flow-shop scheduling. Section 6 includes a numerical illustration example. Section 7 presents a comparison between the solutions from the proposed approach with the others. Finally, concluding remarks are included in Section 8.

\section{Preliminaries}

In order to easily discuss the problem, it recalls basic rules and findings related to fuzzy numbers, pentagonal fuzzy numbers, and arithmetic operations of pentagonal fuzzy numbers and its ranking.

Definition 1 (see [21-24]). A fuzzy set $\widetilde{A}$ a set of real numbers $R$ is called fuzzy if its membership function $\mu_{A}(x): \mathbb{R} \longrightarrow[0,1]$ have the following properties:

(1) $\mu_{\widetilde{A}}(x)$ is an upper semicontinuous membership function

(2) $\widetilde{A}$ is convex fuzzy set, i.e., $\mu_{\tilde{A}}(\delta x+(1-\delta) y) \geq$ $\min \left\{\mu_{\mathcal{A}}(x), \mu_{\mathcal{A}}(y)\right\}$ for all $x, y \in \mathbb{R} ; 0 \leq \delta \leq 1$

(3) $\widetilde{A}$ is normal, i.e., $\exists x_{0} \in \mathbb{R}$ for which $\mu_{\tilde{A}}\left(x_{0}\right)=1$
(4) Supp $(\tilde{A})=\left\{x \in \mathbb{R}: \mu_{\tilde{A}}(x)>0\right\}$ is the support of $\widetilde{A}$, and the closure $\operatorname{cl}(\operatorname{Supp}(\widetilde{A}))$ is compact set

Definition 2 (see $[8,22,23$ to $9,25,26]$ ). A fuzzy number $\widetilde{A}_{P}=\left(a_{1}, a_{2}, a_{3}, a_{4}, a_{5}\right), a_{1} \leq a_{2} \leq a_{3} \leq a_{4} \leq a_{5}$ on $\mathbb{R}$ is said to be a pentagonal fuzzy number if its membership function is

$$
\mu_{A_{P}}= \begin{cases}0, & x<a_{1}, \\ w_{1}\left(\frac{x-a_{1}}{a_{2}-a_{1}}\right), & \text { for } a_{1} \leq x \leq a_{2}, \\ 1-\left(1-w_{1}\right)\left(\frac{x-a_{2}}{a_{3}-a_{2}}\right), & \text { for } a_{2} \leq x \leq a_{3}, \\ 1-\left(1-w_{2}\right)\left(\frac{a_{4}-x}{a_{4}-a_{3}}\right), & \text { for } a_{3} \leq x \leq a_{4}, \\ w_{2}\left(\frac{a_{5}-x}{a_{5}-a_{4}}\right), & \text { for } x=a_{3}, \\ 0, & \text { for } a_{4} \leq x \leq a_{5},\end{cases}
$$

Figure 1 shows the graphical representation of the pentagonal fuzzy number $\widetilde{A}_{P}$.

Definition 3. Let $\widetilde{A}_{P}=\left(a_{1}, a_{2}, a_{3}, a_{4}, a_{5}\right)$ and $\widetilde{B}_{P}=\left(b_{1}, b_{2}\right.$, $b_{3}, b_{4}, b_{5}$ ) be two pentagonal fuzzy numbers and $v \neq 0$. The arithmetic operations on $\widetilde{A}_{P}$ and $\widetilde{B}_{P}$ are

(i) $\widetilde{A}_{P} \oplus \widetilde{B}_{P}=\left(a_{1}+b_{1}, a_{2}+b_{2}, a_{3}+b_{3}, a_{4}+b_{4}, a_{5}+b_{5}\right)$

(ii) $\widetilde{A}_{P} \ominus \widetilde{B}_{P}=\left(a_{1}-b_{5}, a_{2}-b_{4}, a_{3}-b_{3}, a_{4}-b_{2}, a_{5}-b_{1}\right)$

(iii) $\widetilde{A}_{P} \otimes \widetilde{B}_{P}=1 / 5 \gamma_{b}\left(a_{1}, a_{2}, a_{3}, a_{4}, a_{5}\right), \gamma_{b}=\left(b_{1}+b_{2}+\right.$ $\left.b_{3}+b_{4}+b_{5}\right)$

(iv) $\widetilde{A}_{P} \oslash \widetilde{B}_{P}=\left(5 / \gamma_{b}\right)\left(a_{1}, a_{2}, a_{3}, a_{4}, a_{5}\right), 0 \neq \gamma_{b}=$ $\left(b_{1}+b_{2}+b_{3}+b_{4}+b_{5}\right)$

(v) $k \widetilde{A}_{P}= \begin{cases}\left(k a_{1}, k a_{2}, k a_{3}, k a_{4}, k a_{5}\right) m, & k>0, \\ \left(k a_{5}, k a_{4}, k a_{3}, k a_{2}, k a_{1}\right), & k<0\end{cases}$

Definition 4 (see [25]). The ranking function of $\widetilde{A}_{P}=\left(a_{1}\right.$, $\left.a_{2}, a_{3}, a_{4}, a_{5}\right)$ is defined as

$$
R\left(\widetilde{A}_{P}\right)=\int_{0}^{1} 0.5\left[\left(a_{2}-a_{1}\right) \alpha+a_{1},-\left(a_{5}-a_{4}\right) \alpha+a_{5}\right] \mathrm{d} \alpha .
$$

Definition 5. The order of relation between $\widetilde{A}_{P}$ and $\widetilde{B}_{P}$ based on the ranking function is defined as

(i) $\widetilde{A}_{P} \pm \widetilde{B}_{P}$ if and only if $R\left(\widetilde{A}_{P}\right) \geq R\left(\widetilde{B}_{P}\right)$

(ii) $\widetilde{A}_{P} \circ \widetilde{\mathrm{B}}_{\mathrm{P}}$ if and only if $\mathrm{R}\left(\widetilde{\mathrm{A}}_{\mathrm{P}}\right) \leq \mathrm{R}\left(\widetilde{\mathrm{B}}_{\mathrm{P}}\right)$

(iii) $\widetilde{A}_{P} \cong \widetilde{B}_{P}$ if and only if $R\left(\widetilde{A}_{P}\right)=R\left(\widetilde{B}_{P}\right)$ 


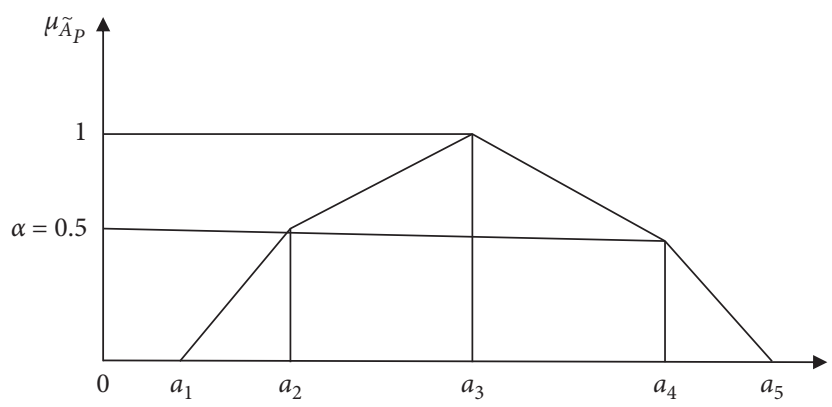

FIgURE 1: Graphical representation of pentagonal fuzzy number [4].

\section{Assumptions, Notations, and Rental Policy}

In this flow-shop scheduling problem, the following assumptions are made.

\subsection{Assumptions}

(i) No job pre-emption is allowed

(ii) Just one function can be performed by the machine

(iii) All jobs are available at the beginning of schedule time

(iv) The initialization times of the machines are negligible

(v) All workers are processed throughout the deterministic phase

(vii) Due dates are pentagonal fuzzy numbers

(viii) The machine may be idle

(viii) The production period is separate from the schedule

(ix) The first machine must be completed to provide a second device with feeding

(x) For each mission, $M$ operations are needed

(xi) Any job must be done as it has begun

3.2. Notations. The following notations are allowed in the flow-shop scheduling problem.

$S_{k}$ : Sequence resulted by applying Johnson's procedure, $k(k=1,2, \ldots, m)$

$M_{j}$ : Machine $j(j=1,2, \ldots, m)$

$\tilde{M}_{P}:$ Machine makes span

$\left(\widetilde{A}_{P}\right)_{i j}$ : Pentagonal fuzzy processing time of ith job on machine $M_{j}, i=1,2, \ldots, n$ and $j=(1,2, \ldots, m)$

$\left(\widetilde{T}_{P}\right)_{i j}\left(S_{k}\right)$ : Completion time of ith job of sequence $S_{k}$ on machine $M_{j}(j=1,2, \ldots, m)$

$\left(\widetilde{I}_{P}\right)_{i j}\left(S_{k}\right)$ : Idle time of machine $M_{j}(j=1,2, \ldots, m)$, for job $i(i=1,2, \ldots, n)$ in the sequence $S_{k}$

$\left(\widetilde{U}_{P}\right)_{j}\left(S_{k}\right)$ : Utilization time of which machine $M_{j}(j=$ $1,2, \ldots, m)$ is required

$\widetilde{R}_{P}\left(S_{k}\right)$ : Total rental cost for the sequence $S_{k}$ for all machines $M_{j}(j=1,2, \ldots, m)$
$\left(\widetilde{C}_{P}\right)_{i}$ : Rental cost of $i$ th machine

$\widetilde{C}_{P}\left(S_{i}\right)$ : Total completion time of the jobs for sequence $i(i=1,2, \ldots, n)$

3.3. Rental Policy. The first machine will be taken on rent in the beginning of the processing of the jobs; the second machine will be taken on rent when the first job is completed on the first machine and transported to the second machine. The third machine will be taken on rent when the first job is completed on the second machine and transported to the third machine and so on.

\section{Problem Formulation}

Assume that job $i(i=1,2, \ldots, n)$ is to be processed on machine $j(j=1,2, \ldots, m)$ in the existence of specified rental policy. Let $\left(\widetilde{A}_{P}\right)_{i j}(i=1,2, \ldots, n ; j=1,2, \ldots, m)$ be the processing time of $i$ th job on $j$ th machine characterized by pentagonal fuzzy numbers. The problem can be formulated as

$$
\begin{aligned}
\min \widetilde{R}_{P}\left(S_{k}\right)= & \sum_{i=1}^{n}\left[\left(\widetilde{A}_{P}\right)_{i 1} \times\left(\widetilde{C}_{P}\right)_{1}+\left(\widetilde{U}_{P}\right)_{2}\left(S_{k}\right) \times\left(\widetilde{C}_{P}\right)_{2}\right. \\
& \left.+\left(\widetilde{U}_{P}\right)_{3}\left(S_{k}\right) \times\left(\widetilde{C}_{P}\right)_{3}\right]
\end{aligned}
$$

which subjects to rental policy $P$.

\section{Solution Method}

In this section, the method to minimize the utilization time and then the rental cost of flow-shop scheduling problem under fuzzy environment is provided in the following steps.

Step 1 : convert the problem into a problem of two machines if one of the following conditions is reached:

(i) $\min \left(\widetilde{A}_{P}\right)_{i 1} \pm \max \left(\widetilde{A}_{P}\right)_{i j}, j=2,3, \ldots, m-1$

(ii) $\min _{i}^{i}\left(\widetilde{A}_{P}\right)_{i m} \pm \max _{i}^{i}\left(\widetilde{A}_{P}\right)_{i j}, j=2,3, \ldots, m-1$

Step 2 : convert this problem to a problem of two machines and add two fictional $\mathrm{H} 1$ and $\mathrm{H} 2$ machines, $\left(\widetilde{H}_{P}\right)_{1}^{i}=\sum_{j=1}^{m-1}\left(\widetilde{A}_{P}\right)_{i j}, i=1,2, \ldots, n$, and $\left(\widetilde{H}_{P}\right)_{2}^{i}=$ $\sum_{j=2}^{m}\left(\tilde{A}_{P}\right)_{i j}, \quad i=1,2, \ldots, n$, where $\left(\tilde{H}_{P}\right)_{1}^{i}$ and 
TABLE 1: Pentagonal fuzzy processing times $\tilde{A}_{P}=\left(a_{1}, a_{2}, a_{3}, a_{4}, a_{5}\right)$.

\begin{tabular}{lccc}
\hline Job & Machine $\mathrm{M}_{1}$ & Machine $\mathrm{M}_{2}$ & ${\text { Machine } \mathrm{M}_{3}}$ \\
\hline 11 & $(2,6,8,10,14)$ & $(2,5,7,8,9)$ & $(1,3,4,5,6)$ \\
22 & $(11,12,13,14,16)$ & $(4,5,6,7,8)$ & $(3,4,5,68)$ \\
33 & $(6,8,10,12,13)$ & $(3,4,5,68)$ & $(2,5,7,8,9)$ \\
4 & $(8,9,11,12,13)$ & $(4,5,6,7,8)$ & $(9,11,12,13,15)$ \\
5 & $(7,9,10,11,13)$ & $(4,5,6,8,10)$ & $(6,8,9,11,12)$ \\
\hline
\end{tabular}

TABle 2: Pentagonal fuzzy processing times for fictions machines $H_{1}$ and $H_{2}$.

\begin{tabular}{lcr}
\hline Job & $H_{1}$ & $H_{2}$ \\
\hline 1 & $(4,11,15,18,23)$ & $(3,8,11,13,15)$ \\
2 & $(15,17,19,21,24)$ & $(7,9,11,13,16)$ \\
3 & $(9,12,15,18,21)$ & $(5,9,12,14,17)$ \\
4 & $(12,14,17,19,21)$ & $(13,16,18,20,23)$ \\
5 & $(11,14,16,19,23)$ & $(10,13,15,19,22)$ \\
\hline
\end{tabular}

TABLE 3: Time in and time out for machines.

\begin{tabular}{lcccccc}
\hline Job & \multicolumn{2}{c}{ Machine 1} & \multicolumn{2}{c}{ Machine 2 } & \multicolumn{2}{c}{ Machine 3} \\
Time in & Time out & Time in & Time out & Time in & Time out \\
\hline 1 & $(0,0,0,0,0)$ & $(8,9,11,12,13)$ & $(8,9,11,12,13)$ & $(12,14,17,19,21)$ & $(12,14,17,19,21)$ & $(21,25,29,32,37)$ \\
2 & $(8,9,11,12,13)$ & $(15,18,21,23,26)$ & $(15,18,21,23,26)$ & $(19,23,27,30,34)$ & $(21,25,29,32,37)$ & $(27,33,38,43,49)$ \\
3 & $(15,18,21,23,26)$ & $(24,30,36,41,47)$ & $(24,30,36,41,47)$ & $(25,30,35,39,44)$ & $(27,33,38,43,49)$ & $(29,38,45,51,58)$ \\
4 & $(24,30,36,41,47)$ & $(39,47,55,62,71)$ & $(39,47,55,62,71)$ & $(36,44,51,58,62)$ & $(36,44,51,58,62)$ & $(39,48,56,64,70)$ \\
5 & $(39,47,55,62,71)$ & $(41,53,63,72,85)$ & $(41,53,63,72,85)$ & $(40,55,66,76,85)$ & $(40,55,66,76,85)$ & $(41,58,70,81,91)$ \\
\hline
\end{tabular}

$\left(\widetilde{H}_{P}\right)_{2}^{i}$ are the fuzzy pentagonal processing time for job $i$ on machines $H_{1}$ and $H_{2}$, respectively.

Step 3 : apply the algorithm introduced by [27] on machines $H_{1}$ and $H_{2}$, to obtain the sequence $S_{k}$.

\section{Numerical Example}

Consider 5 jobs and 3 machines' flow-shop scheduling problem having fuzzy processing times represented by pentagonal fuzzy numbers as in Table 1 . The rental cost per unit time for machines $M_{1}, M_{2}$, and $M_{3}$ are 5, 3, and 4 units, respectively. Our aim is to minimize the total rental cost [28].

Table 1 illustrates the three machines scheduling problem with processing times characterized by pentagonal fuzzy numbers.

(1) For $M_{1}$ : processing time $\min$ is $(2,6,8,10,14)$

(2) For $M_{2}$ : processing time $\max$ is $(2,5,7,8,9)$

(3) For $M_{3}$ : processing time $\min$ is $(1,3,4,5,6)$

Here, all the decision parameters are represented by pentagonal fuzzy numbers.

It is clear that Min time of $M_{1}(2,6,8,10,14) \geqslant$ Max time of $M_{2}(2,5,7,8,9)$. Let $H_{1}$ and $H_{2}$ be two fictitious machine such that

$$
\begin{aligned}
& \left(\widetilde{H}_{P}\right)_{1}^{i}=\sum_{j=1}^{2}\left(\widetilde{A}_{P}\right)_{i j} \\
& \left(\tilde{H}_{P}\right)_{2}^{i}=\sum_{j=2}^{3}\left(\widetilde{A}_{P}\right)_{i j}
\end{aligned}
$$

Then, Table 2 shows the fictitious machine $H_{1}$ and $H_{2}$.

Based on the algorithm introduced by [27], the order of sequencing is $S_{k}=4 \longrightarrow 5 \longrightarrow 3 \longrightarrow 2 \longrightarrow 1$.

Hence, Table 3 provides the time in and out of the machines.

In addition, the times idle and rental costs are calculated in Table 4 . The total elapsed time $=(41,58,70,81,91)$ unit of time.

Thus, the total rental cost is $(205,265,315,360,425) \oplus$ $(-194,-96,63,186,381) \oplus(-152,40,216,260,572)=(-141$, $209,594,806,378)$ units of cost.

\section{Comparative Study}

From Table 5, it is clear that the objective function value resulted from the Sathish and Ganesan algorithm is the same with the proposed approach, and the overall rental costs for the machines resulting from this strategy are clearly lower than those found by Gupta et al. [18]. 


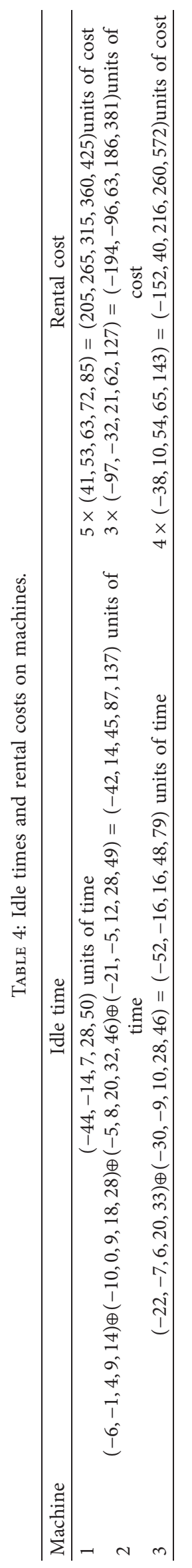


TABLE 5: Comparison of the example results.

\begin{tabular}{lcc}
\hline Proposed approach & $\begin{array}{c}\text { Algorithm by Sathish and Ganesan } \\
{[28]}\end{array}$ & Method by Gupta et al. [18] \\
\hline $\begin{array}{l}\text { Total rental cost equal to }(-141,209,452,806,1378) \\
\text { units of cost }\end{array}$ & $\begin{array}{c}\text { Total rental cost equal to }(452,8,8) \\
\text { units of cost }\end{array}$ & $\begin{array}{c}\text { Total rental cost equal to }(377,613,662) \\
\text { units of cost }\end{array}$ \\
\hline
\end{tabular}

\section{Concluding Remarks}

In this paper, we provided a clear approach for the preparation of the flow-shop problem under fuzzy environment in which processing time of jobs are represented by pentagonal fuzzy numbers. The fuzzy FS scheduling problem was solved without converting the processing time into its equivalent crisp numbers using a robust ranking technique and a fuzzy arithmetic pentagonal fuzzy numbers. The strategy aims to reduce the rental costs of the machine in accordance with the leasing system. The advantage of this approach is more flexible where it allows the decision maker to choose the targets he/she is willing and useful for potential study in widespread fluctuations.

\section{Data Availability}

The data used to support the findings of this study are available from the corresponding author upon request.

\section{Conflicts of Interest}

The authors declare no conflicts of interest.

\section{References}

[1] S. Argawal, A. Gautain, D. K. Chauhan, L. M. Twari, and S. Kapoor, "A flow- shop scheduling problem with transportation time and separated setup time of jobs," Procedia Engineering, vol. 38, pp. 1327-1332, 2012.

[2] B. Vahedi- Nouri, P. Fattahi, R. Tavakkoli- Moghaddam, and R. Ramezanian, "A algorithm for flow shop scheduling problem with consideration of position-based learning effect and multiple availability constraints," International Journal of Advanced Manufacturing Technology, vol. 73, no. 5-8, pp. 601-611, 2014.

[3] T. Ren, M. Guo, L. Lin, and Y. Miao, "A local search algorithm for the flow- shop scheduling problem with release dates," Discrete Dynamics in Nature and Society, vol. 2015, Article ID 320140, 8 pages, 2015.

[4] I. Laribi, F. Yalaoui, F. Belkaid, and Z. Sari, "Heuristics for solving flow shop scheduling problem under resources constraints," IFAC-PapersOnLine, vol. 49, no. 12, pp. 1478-1483, 2016.

[5] M. Yazdani and B. Naderi, "Modeling and scheduling no- idle hybrid flow shop problem," Journal of Optimization in Industrial Engineering, vol. 21, pp. 59-66, 2017.

[6] C. Qu, Y. Fu, Z. Yi, and J. Tan, "Solutions to no- wait flow- shop scheduling problem using the flower pollination algorithm based on the hormone modulation mechanism," Complexity, vol. 2018, Article ID 1973604, 18 pages, 2018.

[7] R. Bellmann and L. Zadeh, "Decision making in a fuzzy environment," Management Science, vol. 17, pp. 141-164, 1970.
[8] D. Dubois and H. Prade, Fuzzy Sets and Systems; Theory and Applications, Academic Press, New York, NY, USA, 1980.

[9] A. Chakraborty, S. P. Mondal, S. Alam et al., "The pentagonal fuzzy number: its different representation, properties, ranking, defuzzification, and application in game problems," Symmetry, vol. 11, pp. 1-31, 2019.

[10] R. R. Yager, "A procedure for ordering fuzzy subsets of the unit interval," Information Sciences, vol. 24, pp. 143-161, 1981.

[11] L. Martin and T. Robert, "Fuzzy scheduling with application to real time system," Fuzzy Sets and Systems, vol. 121, no. 3, pp. 523-535, 2001.

[12] J.-S. Yao and F.-T. Lin, "Constructing a fuzzy flow-shop sequencing model based on statistical data," International Journal of Approximate Reasoning, vol. 29, no. 3, pp. 215-234, 2002.

[13] S. R. Hejari, S. Emami, and A. Arkan, "A Heuristic algorithm for minimizing the expected makes span in two machine flowshops with fuzzy processing time," Journal of Uncertain Systems, vol. 3, no. 2, pp. 114-122, 2009.

[14] S. McCahon and E. S. Lee, "Job sequencing with fuzzy processing times," Computer and Mathematics Applications, vol. 19, no. 7, pp. 294-301, 1990.

[15] H. Ishibuchi and K. H. Lee, "Formulation of fuzzy flow- shop scheduling problem with fuzzy processing time," in Proceedings of the IEEE International Conference on Fuzzy System, pp. 199-205, New Orleans, LA, USA, August 2001.

[16] I. Temiz and S. Erol, "Fuzzy branch- and- bound algorithm for flow shop scheduling," Journal of Intelligent Manufacturing, vol. 15, no. 4, pp. 449-454, 2004.

[17] E. Ignall and L. Schrage, "Application of the branch and bound technique to some flow-shop scheduling problems," Operations Research, vol. 13, no. 3, pp. 400-412, 1965.

[18] D. Gupta, S. Aggarwal, and S. Sharma, "A Fuzzy logic based approach to minimize the rental cost of machines for specially structured three stages flow- shop scheduling," Advances in Applied Science Research, vol. 3, no. 2, pp. 1071-1076, 2012.

[19] E. M. G. Neira, J. R. Montoyao- Torres, and D. Barrera, "Flowshop scheduling problem under uncertainties: review and trends," International Journal of Industrial Engineering Computation, vol. 8, no. 4, pp. 399-426, 2017.

[20] G. M. Komaki, S. Sheikh, and B. Malakooti, "Flow shop scheduling problems with assembly operations: a review and new trends," International Journal of Production Research, vol. 57, no. 10, pp. 2926-2955, 2019.

[21] E. Janaki and A. Mohamed Ismail, "Flow shop scheduling in which processing time connected with probabilities and job delay due to maintenance for $\mathrm{M}^{*} \mathrm{~N}$ machine," Information and Communication Technology for Sustainable Development, vol. 2, pp. 651-657, 2020.

[22] H. A. Khalifa, "On single machine scheduling problem with distinct due dates under fuzzy environment," International Journal of Supply and Operations Management, vol. 7, no. 3, pp. 272-278, 2020.

[23] H. A. Khalifa, S. A. Sultan, and P. Kumar, "Solving constrained flow- shop scheduling problem through multistage 
fuzzy binding approach with fuzzy due dates," Advances in Fuzzy Systems, vol. 2021, Article ID 6697060, 8 pages, 2021.

[24] L. A. Zadeh, "Fuzzy sets," Information and Control, vol. 8, no. 3, pp. 338-353, 1965.

[25] S. Abbasbandy and T. Hajjari, "A new approach for ranking of trapezoidal fuzzy numbers," Computers and Mathematics with Applications, vol. 57, pp. 413-419, 2009.

[26] A. Panda and M. Pal, "A study on pentagonal fuzzy number and its corresponding matrices," Pacific Science Review B: Humanities and Social Sciences, vol. 10, pp. 1-9, 2016.

[27] S. M. Johnson, "Optimal two- and three-stage production schedules with setup times included," Naval Research Logistics Quarterly, vol. 1, no. 1, pp. 61-68, 1954.

[28] S. Sathish and K. Ganesan, "Flow- shop scheduling problem to minimize the rental cost under fuzzy environment," Journal of Natural Sciences Research, vol. 2, no. 10, pp. 62-68, 2012. 